\title{
Outcomes of a superiorly-based pharyngeal flap for the correction of velopharyngeal dysfunction
}

\author{
Yong Woo Lee ${ }^{1}$, \\ Yong Chan $\mathrm{Bae}^{1,2}$, \\ Sang Min Park ${ }^{1}$, \\ Soo Bong Nam ${ }^{1}$, \\ Hyung Joon Seo ${ }^{1}$, \\ Geon Woo Kim ${ }^{1}$ \\ ${ }^{1}$ Department of Plastic and \\ Reconstructive Surgery, Pusan National \\ University School of Medicine, Busan; \\ ${ }^{2}$ Biomedical Research Institute, Pusan \\ National University Hospital, Busan, \\ Korea
}

\begin{abstract}
Background: This study investigated the outcomes of pharyngeal flap surgery in Asian patients with velopharyngeal dysfunction (VPD), with a focus on postoperative improvements in speech articulation and complications. Additionally, this study explored whether the cause of VPD and the timing of surgery affected outcomes in terms of speech improvement.

Methods: A retrospective review was conducted of the medical records of 72 Asian VPD patients who underwent pharyngeal flap surgery performed by a single surgeon from 1996 to 2014. Operative complications were analyzed for all 72 patients, and preoperative and postoperative speech articulation tests were compared for the subset of 43 patients in whom such testing was possible. The relationships of age at surgery and the cause of VPD with the extent of improvement in speech articulation were also investigated.

Results: Nine of the 72 patients (13\%) experienced wound-related complications. Furthermore, 34 patients (47\%) reported persistent snoring lasting for over 3 months, and $16(22 \%)$ reported snoring for over 1 year. Three patients (4\%) underwent reoperation. Speech articulation scores significantly improved postoperatively across all groups. Younger age at surgery was associated with greater improvements in speech outcomes, but no significant relationship was found between the cause of VPD and the extent of the postoperative improvement in speech outcomes. Conclusion: Speech outcomes improved after pharyngeal surgery in Asian patients with VPD. Early surgery was associated with favorable speech outcomes, but no association was found between the cause of VPD and postoperative speech outcomes.
\end{abstract}

Keywords: Cleft palate / Speech / Surgery / Surgical flap / Velopharyngeal Insufficiency

\section{INTRODUCTION}

Velopharyngeal dysfunction (VPD) can be treated through non-surgical and surgical modalities. Surgical options include superiorly-based pharyngeal flaps, palatal lengthening (double

Correspondence: Yong Chan Bae

Department of Plastic and Reconstructive Surgery, Biomedical Research Institute, Pusan National University Hospital, Pusan National University School of Medicine, 179 Gudeok-ro, Seo-gu, Busan 49241, Korea

E-mail: baeyc2@hanmail.net

This work was supported by a 2-year research grant from Pusan National University Hospital.

Received December 3, 2019 / Revised January 19, 2020 / Accepted January 24, 2020 opposing Z-plasty; Furlow palatoplasty), and sphincter pharyngoplasty. The most commonly used surgical technique for treating VPD following cleft palate repair is a posterior pharyngeal flap. Superiorly-based flaps are more effective than inferiorly-based flaps for treating VPD because they can be made longer, whereas the length of inferiorly-based flaps is limited by the presence of an adenoid pad. Nonetheless, postoperative acute or chronic complications may occur, including residual VPD, snoring, obstructive sleep apnea (OSA), and even dehiscence. Most complications are acute, occurring within 24 hours postoperatively [1], and Fraulin et al. [2] showed that the overall complication rate decreased from $19.5 \%$ to $6.3 \%$ during a 
several-year period.

Previous studies have investigated factors related to the effectiveness of VPD treatment with superiorly-based pharyngeal flaps. Specifically, previous research has explored the associations of the cause of VPD and age at surgery with speech outcomes [3-6]. Some studies have reported that age at surgery was not a critical factor, while others have shown that optimal speech improvements were achieved in younger children [5,7].

In this study of Asian VPD patients, the outcomes of superiorly-based pharyngeal flap surgery performed by a single surgeon were analyzed in terms of postoperative speech outcomes and complications. Speech outcomes were compared according to the cause of VPD and age at surgery.

\section{METHODS}

\section{Subjects}

Patients treated for VPD from September 1996 to October 2014 were reviewed, and the subjects of this study comprised $72 \mathrm{pa}-$ tients in whom pharyngeal flap surgery was indicated, as confirmed by preoperative speech articulation testing, nasoendoscopy, and lateral video fluoroscopy. An additional inclusion criterion was at least 6 months of follow-up after pharyngeal flap surgery. Surgical complications were evaluated in all 72 patients, and speech outcomes were analyzed in 43 patients for whom speech articulation testing was possible. This study was conducted after receiving approval from the Institutional Review Board (IRB No. 1907-020-081), and all patients (or their guardians) provided informed written consent.

\section{Complications}

Acute and chronic complications associated with superiorlybased pharyngeal flap surgery were analyzed in all 72 VPD patients. Acute complications included airway, flap, and donor site problems, whereas chronic complications were determined based on examinations assessing snoring and other respiratory difficulties. For snoring, patients were classified according to whether their symptoms lasted for over 3 months and for over 1 year.

\section{Speech articulation testing}

Speech outcomes were evaluated through articulation testing, both preoperatively and at the postoperative follow-up (at 6 months or more). On average, postoperative articulation testing was conducted at 8.5 months after surgery (range, 6-24 months). The simple method of evaluating speech in Korean cleft palate patients developed by Kim et al. [8] was used to score their speech. In this method, a total of 22 items are evaluated (hypernasality, five items; plosives, seven items; fricatives, two items; affricates, three items; laterals, two items; and nasal consonants, three items), with a total possible score of 66 points. Five doctors (including a plastic surgery resident) and five medical school students conducted speech articulation testing. Patients' scores were summed and averaged.

\section{Analysis of speech articulation testing data}

The significance of improvements in speech articulation was determined by analyzing the preoperative and postoperative speech articulation test results using the paired $t$-test. The relationship of the cause of VPD with speech articulation improvement was analyzed using one-way analysis of variance (ANOVA) for five groups (cleft palate, incomplete, cleft palate submucous, cleft lip and palate, complete and idiopathic). The relationship between age at surgery and speech outcomes was also analyzed by one-way ANOVA for four groups (4-5 years, 6-10 years, $11-20$ years, and $\geq 21$ years). The $p$-values were obtained from Mann-Whitney tests conducted as post-hoc tests. This relationship was also quantified by calculating correlation coefficients through linear regression analysis between age at surgery and improvement in the speech articulation test score. Excel 2010 (Microsoft Corp., Redmond, WA, USA) was used for all statistical analyses and $p$-values $<0.05$ were considered to indicate statistical significance except the Mann-Whitney test. $p$ values $<0.0125$ in the Mann-Whitney test using Bonferroni's method were considered to indicate statistical significance.

\section{RESULTS}

\section{Complications}

None of the 72 patients had airway problems immediately after surgery. Nine patients (13\%) experienced acute complications at the flap or donor site, including donor site dehiscence in eight patients and pharyngeal flap dehiscence in one patient. Two patients needed at least 2 weeks for complete wound healing. No patients experienced OSA as a chronic complication. However, 35 patients (48\%) experienced snoring for more than 3 months after surgery, and snoring lasted for more than 1 year in 16 patients $(22 \%)$.

The patient who experienced flap dehiscence underwent reoperation using the same surgical technique. Two patients underwent flap division due to ongoing postoperative discomfort, snoring, and hypernasality.

\section{Changes in speech articulation test scores}

The average age of the 43 patients for whom it was possible to conduct speech articulation testing was $14.1 \pm 11.8$ years (range, 4-52 years). The sex ratio was approximately 1:1 (21 males and 
22 females). Preoperatively, the speech articulation test scores ranged from 37.1 to 63.0 (mean, $52.7 \pm 6.7$ ). Postoperatively, the scores ranged from 42.6 to 66.0 (mean, $59.5 \pm 5.7$ ). An average improvement of 6.8 points was seen in the speech articulation scores, which was statistically significant $(p<0.05)$. However, four had slightly decreased postoperative scores.

Association between the cause of VPD and improvements in speech articulation

Incomplete cleft palate was the most common cause of VPD (18 patients). The mean postoperative improvement in speech articulation was highest in patients with submucous cleft palate (10.7 points), but no statistically significant between group difference was found $(p=0.16)$ (Table 1$)$.

\section{Association between age at surgery and improvements} in speech articulation

Statistically significant differences were found between age groups $(p<0.05)$ (Table 2). The $4-5$ years group and the 6-10 years group exhibited significantly greater improvements in speech articulation testing than the $\geq 21$ years group (MannWhitney test, $p<0.125$ ) (Fig. 1). In addition, age at surgery showed a significant negative correlation with the extent of improvement in speech articulation $(p<0.05)$ (Fig. 2).

\section{DISCUSSION}

Although the success rate of pharyngeal flap surgery for VPD

Table 1. Changes in speech articulation scores according to the cause of VPD

\begin{tabular}{lccc}
\hline Group & No. of patients & \multicolumn{1}{c}{ Mean \pm SD } & $p$-value \\
\hline CP incomplete & 18 & $6.29 \pm 6.08$ & 0.16 \\
CP submucous & 8 & $10.72 \pm 7.45$ & \\
CLP complete & 11 & $6.46 \pm 5.74$ & \\
Idiopathic & 6 & $3.63 \pm 2.16$ & \\
\hline
\end{tabular}

VPD, velopharyngeal dysfunction; SD, standard deviation; CP, cleft palate; CLP, cleft lip and palate.

a)Statistical significance was tested by one-way analysis of variances among groups.

Table 2. Change in speech articulation scores by age group

\begin{tabular}{cccc}
\hline Group & No. of patients & Mean \pm SD & $p$-value a) $^{\text {a }}$ \\
\hline Age (yr) & & & $<0.05$ \\
$4-5$ & 12 & $12.08 \pm 5.65$ & \\
$6-10$ & 11 & $6.94 \pm 5.67$ & \\
$11-20$ & 7 & $6.16 \pm 5.95$ & \\
$\geq 21$ & 13 & $2.12 \pm 2.06$ & \\
\hline
\end{tabular}

$\mathrm{SD}$, standard deviation.

a) Statistical significance was tested by one-way analysis of variance among groups. treatment is high, several complications can take place. Respiratory difficulties and flap or donor site dehiscence are examples of acute complications, most of which occur within 24 hours postoperatively. Bleeding, alterations in airway physiology and swelling can cause perioperative airway obstruction, resulting in severe respiratory difficulties that require prompt attention [9]. All patients who experience such complications must receive satisfactory monitoring [9]. We performed real-time saturation monitoring of patients for 24 hours postoperatively to monitor them for the possibility of airway obstruction. Mild breathing difficulties were observed in some patients, but none

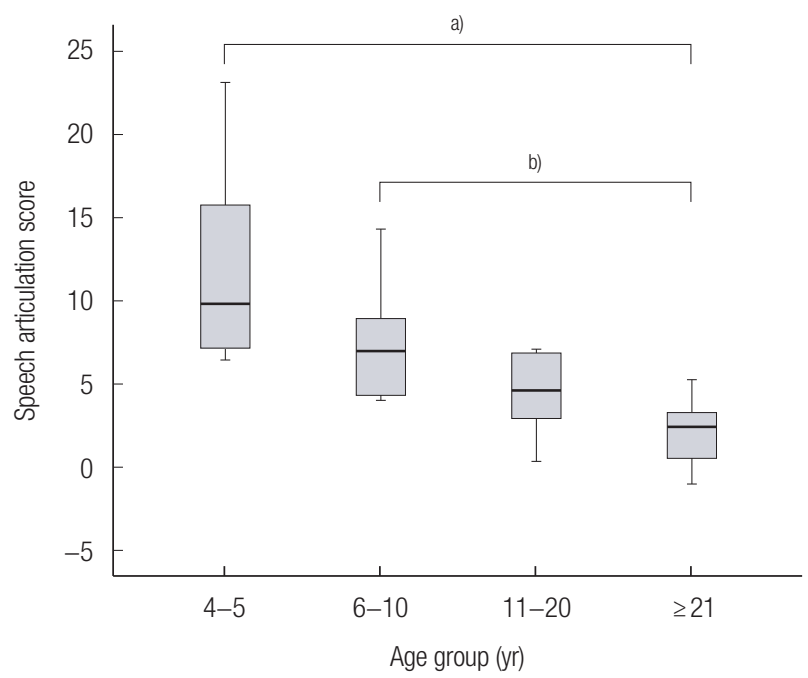

Fig. 1. Comparison of change in speech articulation scores between age groups. ${ }^{\text {a) }}$ The $4-5$ years group exhibited significantly greater improvements in speech articulation testing than the $\geq 21$ years group (Mann-Whitney test, $p<0.125$ ). ${ }^{\text {b) }}$ The $6-10$ years group exhibited significantly greater improvements in speech articulation testing than the $\geq 21$ years group (Mann-Whitney test, $p<0.125$ ).

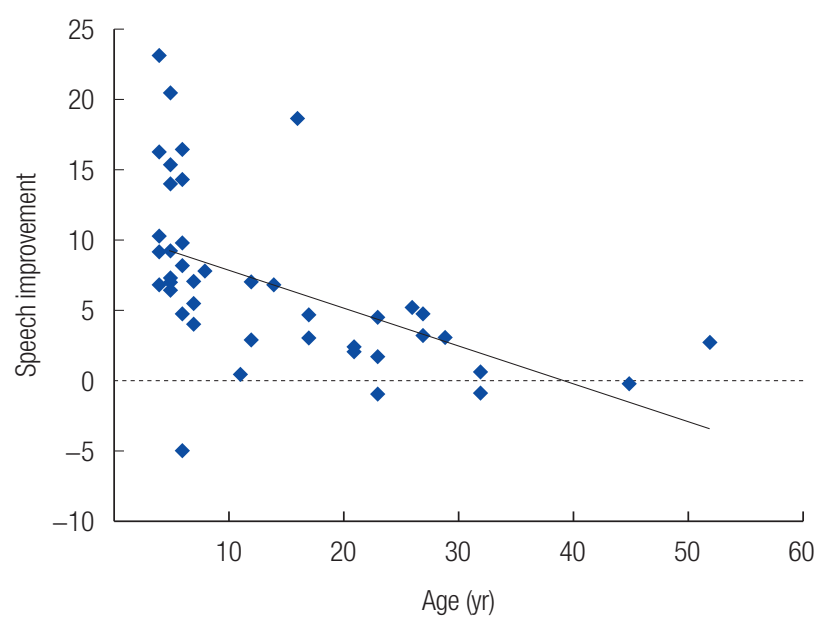

Fig. 2. Speech improvement by age. Speech improvement by age at the time of surgery. Age was negatively correlated with speech improvement $(p<0.05)$. 
had severe respiratory difficulties requiring intubation.

Few studies have investigated flap dehiscence after superiorlybased pharyngeal flap surgery. A study reported complete or partial separation of the flap from the palate in up to $7 \%$ of cases [10]. Studies have also reported that approximately 3\%-20\% of flaps required revision surgery due to dehiscence [1,11-13]. One patient in our study underwent postoperative endotracheal intubation and was transferred to the intensive care unit due to unanticipated arrhythmia during pharyngeal flap surgery. That patient underwent revision surgery using the same technique.

Chronic complications that occur after pharyngeal flap surgery include OSA and hypernasal speech. OSA has been reported to occur in up to $90 \%$ of cases after pharyngeal flap surgery; moreover, since it can potentially cause pulmonary hypertension or right ventricular hypertension, it should be considered as an important complication of pharyngeal flap surgery [1,14-17]. Peat et al. [16] reported that $51 \%$ of patients experienced snoring after pharyngeal flap surgery. Two patients in our study underwent a flap division operation due to ongoing discomfort, snoring, and hypernasality caused by persistent nasal obstruction. These patients underwent superiorly-based pharyngeal flap surgery at 10 and 16 years of age, respectively, and complained of persistent nasal obstruction because they perceived the flap as a foreign object. In these patients, flap division surgery successfully resolved their nasal obstruction, and the improvements in speech outcomes that took place after pharyngeal flap surgery were maintained after the division procedure. This may have been because the residual bulk left in the posterior pharyngeal wall after flap division served as a pad to help with velopharyngeal closure, or as a result of the adaptive speech mechanism that the patient learned when the flap was in place [16].

The cause of VPD and the timing of surgery can be considered as possible factors affecting the outcomes of superiorlybased pharyngeal flap surgery in patients with VPD. Some studies have found that the cause of VPD did not significantly affect the extent of improvements in speech articulation after superiorly-based pharyngeal flap surgery $[4,6]$. Likewise, our study showed postoperative improvements in speech articulation for each cause of VPD, but no significant difference between groups was observed.

Several studies have sought to determine the impact of the timing of surgery on the outcomes of VPD treatment. Some studies have shown that appropriate timing of surgery was associated with a positive impact on improvements in the speech articulation of VPD patients $[5,6,18]$. However, other studies have shown little effect [3,4]. Dorf and Curtin [19] reported a significant difference in postoperative speech abnormalities in cleft palate patients around 1 year of age. In our study, performing pharyngeal flap surgery at a younger age was associated with greater improvements in speech articulation, supporting other studies that have presented positive reports of performing early surgery in VPD patients, before language development. Early surgery may be especially advantageous for improving postoperative speech articulation in light of recent developments that have shortened the operative time for surgery to treat VPD.

VPD patients who are older than 5 years and have well-developed language habituate to pathological malarticulation that compensates for hypernasality, which is caused by their inability to close the velopharynx. Therefore, delaying surgery for VPD might make the development of malarticulation more likely.

Superiorly-pharyngeal flap surgery is one of many ways to treat VPD. Acute and chronic complications may occur after superiorly-based pharyngeal flap surgery, but most such complications resolve without significant sequelae. In this study, we investigated the cause of VPD and the timing of surgery as factors that may affect the outcomes of superiorly-based pharyngeal flap surgery in VPD patients. Significant postoperative improvements in speech outcomes were observed, but with no significant difference according to the cause of VPD, consistent with previous studies. We also investigated the most appropriate timing of surgery for VPD patients, and found that performing surgery at a younger age led to better speech outcomes. In addition, the superiorly-based pharyngeal flap was a successful way to improve speech outcomes in VPD patients, especially those who were younger.

Potential limitations of this study include the fact that it analyzed a small number of patients, especially given the lack of other studies in the literature on additional factors that may influence speech outcomes after superiorly-based pharyngeal flap surgery. Nevertheless, our study is noteworthy in that it makes a novel contribution to the relatively sparse literature on superiorly-based pharyngeal flap surgery in patients with VPD.

\section{NOTES}

\section{Conflict of interest}

No potential conflict of interest relevant to this article was reported.

\section{Ethical approval}

The study was approved by the Institutional Review Board of Pusan National University Hospital (IRB No. 1907-020-081) 
and performed in accordance with the principles of the Declaration of Helsinki. Written informed consent was obtained.

\section{ORCID}

Yong Woo Lee https://orcid.org/0000-0001-8088-822X

Yong Chan Bae https://orcid.org/0000-0002-0268-4667

Sang Min Park https://orcid.org/0000-0001-5884-0983

Soo Bong Nam https://orcid.org/0000-0003-2718-2230

Hyung Joon Seo https://orcid.org/0000-0003-1111-492X

Geon Woo Kim https://orcid.org/0000-0002-5467-9480

\section{REFERENCES}

1. Valnicek SM, Zuker RM, Halpern LM, Roy WL. Perioperative complications of superior pharyngeal flap surgery in children. Plast Reconstr Surg 1994;93:954-8.

2. Fraulin FO, Valnicek SM, Zuker RM. Decreasing the perioperative complications associated with the superior pharyngeal flap operation. Plast Reconstr Surg 1998;102:10-8.

3. Becker DB, Grames LM, Pilgram T, Kane AA, Marsh JL. The effect of timing of surgery for velopharyngeal dysfunction on speech. J Craniofac Surg 2004;15:804-9.

4. Seyfer AE, Prohazka D, Leahy E. The effectiveness of the superiorly based pharyngeal flap in relation to the type of palatal defect and timing of the operation. Plast Reconstr Surg 1988; 82:760-4.

5. Leanderson R, Korlof B, Nylen B, Eriksson G. The age factor and reduction of open nasality following superiorly based velopharyngeal flap operation in 124 cases. Scand J Plast Reconstr Surg 1974;8:156-60.

6. Moll KL, Huffman WC, Lierle DM, Smith JK. Factors related to the success of pharyngeal flap procedures. Plast Reconstr Surg 1963;32:581-8.

7. Van Demark DR, Hardin MA. Longitudinal evaluation of articulation and velopharyngeal competence of patients with pharyngeal flaps. Cleft Palate J 1985;22:163-72.

8. Kim JH, Bae YC, Hwang SM, Jeon JY. Simple method of speech evaluation in the Korean patient with cleft palate. J Korean Soc Plast Reconstr Surg 1999;26:858-65.
9. Wong KWY, Klaiman PG, Forrest CR. Posterior pharyngeal flaps. In: Losee JE, Kirschner RE, editors. Comprehensive cleft care. New York: McGraw-Hill Medical; 2009. p. 649-64.

10. Graham WP 3rd, Hamilton R, Randall P, Winchester R, Stool S. Complications following posterior pharyngeal flap surgery. Cleft Palate J 1973;10:176-80.

11. Dailey SA, Karnell MP, Karnell LH, Canady JW. Comparison of resonance outcomes after pharyngeal flap and furlow double-opposing Z-plasty for surgical management of velopharyngeal incompetence. Cleft Palate Craniofac J 2006;43:38-43.

12. Katzel EB, Shakir S, Naran S, MacIsaac Z, Camison L, Greives $\mathrm{M}$, et al. Speech outcomes after clinically indicated posterior pharyngeal flap takedown. Ann Plast Surg 2016;77:420-4.

13. Agarwal T, Sloan GM, Zajac D, Uhrich KS, Meadows W, Lewchalermwong JA. Speech benefits of posterior pharyngeal flap are preserved after surgical flap division for obstructive sleep apnea: experience with division of 12 flaps. J Craniofac Surg 2003; 14:630-6.

14. Sirois M, Caouette-Laberge L, Spier S, Larocque Y, Egerszegi EP. Sleep apnea following a pharyngeal flap: a feared complication. Plast Reconstr Surg 1994;93:943-7.

15. Ysunza A, Garcia-Velasco M, Garcia-Garcia M, Haro R, Valencia M. Obstructive sleep apnea secondary to surgery for velopharyngeal insufficiency. Cleft Palate Craniofac J 1993;30:38790.

16. Peat BG, Albery EH, Jones K, Pigott RW. Tailoring velopharyngeal surgery: the influence of etiology and type of operation. Plast Reconstr Surg 1994;93:948-53.

17. Liao YF, Chuang ML, Chen PK, Chen NH, Yun C, Huang CS. Incidence and severity of obstructive sleep apnea following pharyngeal flap surgery in patients with cleft palate. Cleft Palate Craniofac J 2002;39:312-6.

18. Meek MF, Coert JH, Hofer SO, Goorhuis-Brouwer SM, Nicolai JP. Short-term and long-term results of speech improvement after surgery for velopharyngeal insufficiency with pharyngeal flaps in patients younger and older than 6 years old: 10-year experience. Ann Plast Surg 2003;50:13-7.

19. Dorf DS, Curtin JW. Early cleft palate repair and speech outcome. Plast Reconstr Surg 1982;70:74-81. 